\title{
Sozialmedizinische Aus-, Fort- und Weiterbildung
}

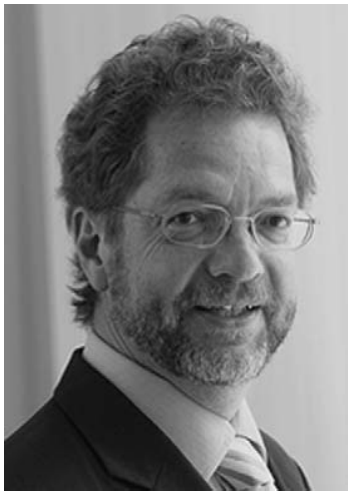

Dr. Matthias Mohrmann

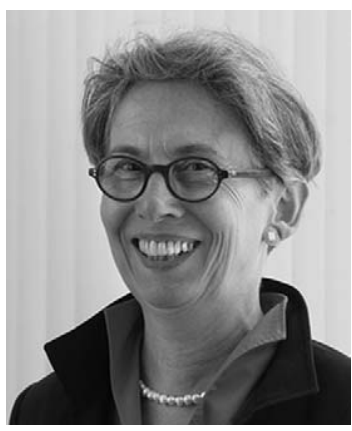

Dr. Elisabeth Nüchtern

Bibliografie

DOI http://dx.doi.org/

$10.1055 / \mathrm{s}-0030-1263133$

Gesundheitswesen 2010;

72: 691

(c) Georg Thieme Verlag KG

Stuttgart · New York

ISSN 0941-3790

Korrespondenzadresse

PD Dr. M. Mohrmann

Leitender Arzt

Medizinischer Dienst der

Krankenversicherung

Baden-Württemberg

Ahornweg 2

77933 Lahr

matthias.mohrmann@

mdkbw.de

\section{Dr. E. Nüchtern}

Leiterin Fachbereich Allgemeine

Sozialmedizin

Medizinischer Dienst der

Krankenversicherung

Baden-Württemberg

Ahornweg 2

77933 Lahr

elisabeth.nuechtern@

mdkbw.de
Die Ausbildung im Medizinstudium ist in Deutschland ebenso wie die ärztliche Weiterbildung fokussiert auf die qualitativ hochwertige Behandlung des einzelnen Patienten. Dabei ist der Ärzteschaft bewusst, dass sie nicht nur dem einzelnen Patienten verpflichtet ist. Die (Muster-) Berufsordnung für die deutschen Ärztinnen und Ärzte (Stand 2006) nennt in $\S 1$ explizit die Verpflichtung der Ärzte gegenüber dem einzelnen Patienten und gegenüber der Bevölkerung. Aus ethischen Gründen ist es auch für den einzelnen Arzt geboten, mit den begrenzten verfügbaren Ressourcen einen möglichst großen gesundheitlichen Nutzen zu erzielen. Zur ärztlichen Verantwortung gegenüber der Bevölkerung gehört die Aufgabe, zum Erhalt der Funktionsfähigkeit unseres Gesundheitswesens insgesamt beizutragen; stellt dieses doch die strukturellen, organisatorischen wie finanziellen Voraussetzungen einer umfassenden guten medizinischen Versorgung bereit. Dabei kommt der Sozialmedizin und den praktisch tätigen Sozialmedizinerinnen und Sozialmedizinern ein wichtiger Part zu.

Sozialmedizin blickt über den einzelnen Patienten hinaus auf den Kontext von Gesundheit und Krankheit. Sie ist ein „Teilgebiet der Medizin, das die Wechselwirkungen zwischen Krankheit und Gesundheit, Individuum und Gesellschaft sowie die Organisationsstrukturen des Gesundheitswesens und des medizinischen Versorgungssystems analysiert, beschreibt und Strategien ... entwickelt“. Dabei verfolgt die Sozialmedizin „einen über die Individualmedizin hinausgehenden, bevölkerungsbezogenen Ansatz“ („Pschyrembel Sozialmedizin“) Auf der Grundlage dieses Selbstverständnisses ergeben sich für die Sozialmedizin spezifische Handlungsfelder und Schwerpunktbereiche. Aufgabe der praktischen Sozialmedizin ist es, wissenschaftliche und einzelfallbezogene Aspekte innerhalb einer angewandten Gesundheitswissenschaft zu integrieren.

Angesichts der Herausforderungen für das Gesundheitswesen durch den demografischen Wandel in Verbindung mit Innovationen in der Medizin, die zu expandierenden Ausgaben führen, erscheint eine solche sozialmedizinische Perspektive dringend geboten.

Werden die Inhalte der Sozialmedizin auch entsprechend in der ärztlichen Aus-, Fort- und Weiterbildung verbreitet? Junge Ärzte, „Berufseinsteiger“, die gerade das Medizinstudium beendet und ihre Weiterbildung begonnen haben, gaben eine negative Antwort auf diese Frage. Sie empfinden laut einer im Deutschen Ärzteblatt 2010 veröffentlichten Umfrage zu rund $60 \%$ im Medizinstudium Defizite hinsichtlich der Ausbildung in puncto Sozialmedizin und Rehabilitation. Trotz dieses beschriebenen Defizits fordern allerdings nur $10-20 \%$ von ihnen eine intensivere Ausbildung in diesem Bereich.

Eine Verbesserung der sozialmedizinischen Ausbildung ist offensichtlich erforderlich, um dem umfassenden Anspruch der Berufsordnung der Ärzte gerecht zu werden. Ausgehen könnte eine Verbesserung der Lehre in der Sozialmedizin von den Universitäten. Einzelne Hochschullehrer sind in der sozialmedizinischen Ausbildung der Medizinstudenten hoch engagiert. Die Sozialmedizin hat jedoch kein einheitliches universitäres Profil. Sie ist nicht an allen medizinischen Fakultäten eigenständig oder mit sozialmedizinischen Lehraktivitäten erkennbar. Da nicht überall in Studium und Weiterbildung eine ausreichende konzeptionelle Grundlage der Sozialmedizin vermittelt wird, überrascht es nicht, dass das Verständnis der klinisch tätigen Ärzte insgesamt für das so wichtige interdisziplinäre Grundlagenfach Sozialmedizin zum Teil begrenzt bleibt und dass im alltäglichen Handeln der Ärzte eine Anwendung sozialmedizinischen Wissens häufig nicht erkennbar wird.

Es muss realistischerweise berücksichtigt werden, dass der Impuls zum Medizinstudium und zur Ausübung des Arztberufes das individuelle Helfen und Heilen wollen ist. In der frühen und mittleren Phase der medizinischen Ausbildung ist die Aufnahmebereitschaft für das stark vernetzende und komplexe sozialmedizinische Wissen deshalb oft auch begrenzt. Umso wichtiger ist eine starke Repräsentanz des Faches Sozialmedizin in der späten universitären Ausbildung und der ärztlichen Fort- und Weiterbildung. Und angesichts des offenbar begrenzten Interesses der Berufseinsteiger ist auch die Förderung methodischer und sozialer Kompetenz der Lehrenden von nicht zu unterschätzender Bedeutung.

Angesichts der Herausforderungen an unser Gesundheitssystem ist eine bessere Verankerung der Sozialmedizin in Studium, Fort- und Weiterbildung von hoher Bedeutung. Die Zeitschrift „Das Gesundheitswesen“ möchte ihren Teil zur sozialmedizinischen Fortbildung beitragen. In der vorliegenden Ausgabe beschäftigen sich die Autoren deshalb auch mit Schlüsselelementen einer neuen Lehr- und Lernkultur.

Es ist lange her, seit Tolstoi formulierte „... zu wissen, was man wissen muss und was man nicht zu wissen braucht, und was man zuerst wissen muss und was später“ (Tagebücher, 1901). 